\title{
Febrile seizure secondary to opioid and benzodiazepine withdrawal in post-operative airway pediatric surgery: a case report
}

\author{
Muhamad Rafiqi Hehsan ${ }^{1,2}$, Wan Fadzlina Wan Muhd Shukeri ${ }^{1}$
}

1. Department of Anaesthesiology \& Intensive Care, School of Medical Sciences, Health Campus, University of Sains Malaysia, 16150 Kubang Kerian, Kelantan, Malaysia.

2. Faculty of Medicine and Health Sciences, Universiti Sains Islam Malaysia, Bandar Baru Nilai, 71800, Nilai, Negeri Sembilan, Malaysia

Correspondence: Dr. Wan Fadzlina Wan Muhd Shukeri; E-mail: wfadzlina@usm.my; Phone: +60129879150

\section{Abstract}

The sedative-analgesic drugs frequently are used to relieve pain and anxiety of patients in intensive care units (ICU). An ideal agent would be rapidly metabolized, and facilitate desirable level of sedation without compromising hemodynamic and respiratory function. This makes opioids and benzodiazepines to be the most commonly prescribed drugs in intensive care. However, weaning off from these drugs may be associated with withdrawal effects. We present a case report of febrile seizure following airway pediatric surgery and opiate and benzodiazepine discontinuation. The weaning off process from these drugs should be meticulously done especially in pediatric age group to avoid subsequent complications.

Key words: Febrile seizure; Opioids; Benzodiazepine; Withdrawal; Post-operative; Airways

Abbreviations: PICU - Pediatric intensive care unit; IWS - latrogenic withdrawal syndrome

Citation: Hehsan MR, Shukeri WFWM. Febrile seizure secondary to opioid and benzodiazepine withdrawal in postoperative airway pediatric surgery: a case report. Anaesth. pain intensive care 2021;25(6):804-806: DOI:

10.35975/apic.v25i6.1709

Received: September 10, 2021, Reviewed: September 18, 2021, Accepted: October 25, 2021

\section{Introduction}

Optimal management of sedation and analgesia provides comfort and security for critical care patients, makes it easier to apply support measures, and allows for a more holistic approach to medical care, while lowering the risk of complications and improving patient outcomes. ${ }^{1}$ The opioids and benzodiazepine groups seem to be the most commonly prescribed drugs in intensive care. ${ }^{1}$ Although both pharmacological categories have clear benefits for critical patients, but they also cause tolerance and physical dependence, necessitating increased dosage and prolonged infusions to obtain the desired effects and avoid the withdrawal phenomenon. ${ }^{2}$

In the pediatric intensive care unit (PICU), the incidence of the iatrogenic withdrawal syndrome (IWS) is comparable, reaching $50 \%$ in patients receiving infusions lasting more than $24 \mathrm{~h}^{3}$

\section{Case Report}

We report the case of an 11 months old baby girl, who was electively admitted for marsupialization of left aryepiglottic cyst under general anesthesia. She suffered from recurrent left aryepiglottic fold cyst formation with mild laryngomalacia. This was her third operative procedure and previous two operations had been successfully carried out. She had a history of noisy breathing (low pitch sound) since birth, which was most noticeable while she was lying supine, crying or while being fed. The baby had a weak cry and occasional feeding interruptions, but was usually able to tolerate breast feeding effectively. Aside from that, there was no history of cyanosis or trauma. Antenatal period had been uneventful and she was born full-term via normal vaginal delivery. Her birth weight was $2.9 \mathrm{~kg}$ and currently weighed $7.5 \mathrm{~kg}$. Family history was irrelevant as there 
was no history of epilepsy or childhood seizures in the family.

The baby successfully underwent marsupialization of left aryepiglottic cyst, and was admitted to surgical intensive care unit (SICU) for postoperative monitoring and weaning. Medication administered in SICU included infusion of midazolam $1-4 \mu \mathrm{g} / \mathrm{kg} / \mathrm{min}$ and fentanyl 5-10 $\mu \mathrm{g} / \mathrm{kg} / \mathrm{h}$, as well as dexamethasone $3.75 \mathrm{mg}$ three times a day. On the first postoperative day, the baby developed enlargement of the tongue and submandibular fullness. Thus, she was proceeded with urgent computed tomography (CT) scan of the neck. CT scan revealed diffuse edema of her tongue, nasopharynx, oropharynx, and larynx, while the trachea and neck vessels were clear. Edema appeared to be present in the submental, submandibular, and adjacent structures of the oral cavity. There was no focal collection or acute hemorrhage seen and the neck circumference was $24 \mathrm{~cm}$. The baby was kept intubated and sedated at that time.

Sedation was tapered off on the postoperative day four, and the baby was extubated and put on a high flow nasal cannula. Post-extubation, there was no stridor or desaturation. Arterial blood gas analysis revealed good oxygenation with no acidosis. Hence, intravenous (IV) dexamethasone was continued for five days and in. Augmentin ${ }^{\circledR} \quad$ (amoxicillin and potassium clavulanate injection) was given IV empirically. At postoperative day six, the child was noted to be less active, stared, not focused and had reduced crying despite being already off-sedation for two days. She then had generalized tonic clonic seizure involving both upper and lower limbs as well as drooling of saliva. The seizure stopped once inj. diazepam was administered intravenously. The baby had a spike of body temperature $38^{\circ} \mathrm{C}$, despite all the blood tests showing being within normal limits. She was suspected to be suffering from meningitis and given cefotaxime and c-penicillin, as well as phenytoin intravenously.

She had two more seizures on the same day, prompting the addition of levetiracetam IV as an antiepileptic. The next day, an urgent CT scan of the brain and electroencephalogram (EEG) test were performed. The results of both tests did not detect any abnormality. The case was subsequently referred to our pediatric neurology team and it was diagnosed as neurological sequel postoperatively, most likely due to opioid or benzodiazepine withdrawal along with nosocomial infection. The Withdrawal Assessment Tool (WAT-1), on the other hand, had a score of zero. Antibiotics were changed to ceftriaxone sodium and metronidazole. After that, there was no seizure was observed. On 12th postoperative day, the baby was discharged home in good physical condition and was prescribed levetiracetam and phenytoin syrup.

\section{Discussion}

Febrile seizures occur in up to $10 \%$ of children under the age of five, and are most common in children aged six months to three years with a peak incidence for the age of around 18 months. ${ }^{4}$ There are no major differences between the genders, but most of the children have a positive family history; the upper respiratory infection being the most common cause. ${ }^{5}$ In our case, there was no seizure history in the family of that 11 months old baby girl. This age may predispose the baby to seizures, regardless of any precipitating condition, which we assume in our instance to be opioid and benzodiazepine withdrawal.

The use of opioids and benzodiazepines to provide children with effective relief of pain and anxiety is a standard practice in the pediatric intensive care unit (PICU) setting. ${ }^{2}$ The most commonly used benzodiazepines for sedation in PICU are midazolam, lorazepam, and diazepam, whereas opioids are commonly used as analgesics for relief of the severe pain. ${ }^{6}$ Analgesia and sedation are frequently necessary for patient's comfort during mechanical ventilation, essential to blunt physiological stress responses and lower the mortality and morbidity risk in a very critically ill patient. Unfortunately, the use of these medications for more than a few days can develop tolerance, which requires higher doses to achieve the same effects, and physical dependency, in which continual drug administration is required to avoid withdrawal syndrome.

Withdrawal syndrome is the term used for a characteristic pattern of unpleasant signs and symptoms that typically follow abrupt cessation of drugs with central nervous system depressant effects. ${ }^{2}$ This condition can affect up to $20 \%$ of children who have been exposed, and are linked to the duration of the infusion and the total dosage. In a review of recent literature, fifty-one symptoms have been described and convulsions are one of those reported. $^{7}$ Sury et al. (1989) reported three cases of children, who received mechanical ventilation for pneumonia and severe asthmatic attacks, suffered from similar signs after they were weaned off. The age range of the children varies from 4 to $12 \mathrm{y}$. Midazolam and morphine are the drugs of choice for sedation and analgesia in all cases. ${ }^{8}$ We consider our case to be unique as it occurred during post-operative period after airway surgery on an 11 month old child. The drug of choice was midazolam and fentanyl. We chose fentanyl because it is more potent, has a faster onset of action, and shorter halflife compared with morphine. ${ }^{9}$

Prompt recognition and management are critical to avoid further associated complications, following weaning of opioids and benzodiazepines in ICU post-operative. There are some validated clinical tools to assess the withdrawal syndrome in PICUs. Three assessment scales 
were identified in a recent systematic review; e.g., WAT1, Sophia Observation Withdrawal Symptoms, and Opioid and Benzodiazepine Withdrawal Score. ${ }^{3}$ The WAT-1 was used in our case, and a score of zero was determined. The lower the score is, the fewer will be the withdrawal symptoms, which was atypical in our case.

Management for withdrawal syndrome in PICUs is complex as therapies are heterogeneous. A recent systematic review identified dexmedetomidine, methadone, and clonidine as possibilities for the treatment and prevention of iatrogenic withdrawal syndrome in PICUs. Drugs such as phenobarbital decrease deprivation symptoms that are resistant to other treatments. $^{3}$

\section{Conclusion}

Withdrawal from opioids and benzodiazepines in pediatric patients during the post-operative period may result in potentially fatal consequences if not promptly managed. Early recognition by ensuring adequate staff training in the use of validated scales will facilitate the assessment of the iatrogenic withdrawal syndrome and subsequently early therapeutic intervention with better prognosis.

\section{Conflict of interest}

None declared by the authors.

\section{Funding statement}

No external funding was involved in the preparation of this case report.

\section{Authors' contribution}

MRH: Conducted the case, literature review, manuscript writing

WFWMS: Supervision of the case, review of the manuscript

\section{References}

1. Celis-Rodríguez $\mathrm{E}$, Birchenall $\mathrm{C}$, de la Cal MÁ, Castorena Arellano $G$, Hernández $A$, Ceraso $D$, et al. Clinical practice guidelines for evidence-based management of sedoanalgesia in critically ill adult patients. Med Intensiva. 2013 Nov;37(8):519-74. [PubMed] DOI: 10.1016/j.medine.2013.04.002

2. Franck LS, Naughton I, Winter I. Opioid and benzodiazepine withdrawal symptoms in paediatric intensive care patients. Intensive Crit Care Nurs. 2004;20(6):344-51. [PubMed] DOI: 10.1016/j.iccn.2004.07.008

3. Ávila-Alzate JA, Gómez-Salgado J, Romero-Martín M, MartínezIsasi S, Navarro-Abal Y, Fernández-García D. Assessment and treatment of the withdrawal syndrome in paediatric intensive care units: Systematic review. Medicine (Baltimore). 2020;99(5):e18502. DOI: 10.1097/MD.0000000000018502

[PubMed]

4. Leung AK, Robson WL. Febrile seizures. J Pediatr Health Care. 2007 Jul-Aug;21(4):250-5.

DOI: 10.1016/j.pedhc.2006.10.006

5. Esmaili Gourabi H, Bidabadi E, Cheraghalipour F, Aarabi Y, Salamat F. Febrile Seizure: Demographic features and causative factors. Iran J Child Neurol. 2012;6(4):33-37. [PubMed]

6. Playfor SD. Analgesia and sedation in critically ill children. Continuing Edu Anaesth Crit Care Pain. 2008;8(3):9094. DOI: 10.1093/bjaceaccp/mkn011

7. Giles B. Opioid and benzodiazepine withdrawal syndromes in the paediatric intensive care unit: a review of recent literature. Nurs Crit Care. 2009;14(1):26-37. [PubMed] DOI: 10.1111/j.14785153.2008.00311.x

8. Sury MR, Billingham I, Russell GN, Hopkins CS, Thornington R, Vivori E. Acute benzodiazepine withdrawal syndrome after midazolam infusions in children. Crit Care Med. 1989;17(3):3012. [PubMed] DOI: 10.1097/00003246-198903000-00027

9. Garimella V, Cellini C. Postoperative pain control. Clin Colon Rectal Surg. 2013;26(3):191-196. [PubMed] DOI: 10.1055/s0033-1351138 